\title{
Mesoscopic cross-film cryotrons: Vortex trapping and dc-Josephson-like oscillations of the critical current
}

\author{
A. Yu. Aladyshkin, ${ }^{1,2}$ G. W. Ataklti, ${ }^{1}$ W. Gillijns, ${ }^{1}$ I. M. Nefedov,${ }^{2}$ I. A. Shereshevsky, ${ }^{2}$ A. V. Silhanek, ${ }^{1}$ J. Van de Vondel, ${ }^{1}$ \\ M. Kemmler, ${ }^{3}$ R. Kleiner, ${ }^{3}$ D. Koelle, ${ }^{3}$ and V. V. Moshchalkov ${ }^{1}$ \\ ${ }^{1}$ INPAC - Institute for Nanoscale Physics and Chemistry, K.U. Leuven, Celestijnenlaan 200D, B-3001 Leuven, Belgium \\ ${ }^{2}$ Institute for Physics of Microstructures RAS, RU-603950 Nizhny Novgorod, GSP-105, Russia \\ ${ }^{3}$ Physikalisches Institut - Experimentalphysik II and Center for Collective Quantum Phenomena, Universität Tübingen, \\ Auf der Morgenstelle 14, D-72076 Tübingen, Germany
}

(Received 18 January 2011; revised manuscript received 15 February 2011; published 13 April 2011)

\begin{abstract}
We investigate theoretically and experimentally the transport properties of a plain Al superconducting strip in the presence of a single straight current-carrying wire, oriented perpendicular to the superconducting strip. It is well known that the critical current of the superconducting strip, $I_{c}$, in such a cryotron-like system can be tuned by changing the current in the control wire, $I_{w}$. We demonstrated that the discrete change in the number of the pinned vortices/antivortices inside the narrow and long strip nearby the current-carrying wire results in a peculiar oscillatory dependence of $I_{c}$ on $I_{w}$.
\end{abstract}

DOI: 10.1103/PhysRevB.83.144509

PACS number(s): 74.25.F-, 74.25.Sv, 74.78.Na

\section{INTRODUCTION}

The original idea to control the resistance of a long superconducting ( $\mathrm{S}$ ) wire by means of a magnetic field generated by a coil wound locally over the wire, was proposed by Buck in $1956 .{ }^{1}$ If the magnetic field inside the solenoid with permanent driving current exceeds the critical field of the central type-I superconducting wire, superconductivity will be completely suppressed and this device (cryotron) will be switched from a low-resistive state to a high-resistive state. Further investigations in the $1960 \mathrm{~s}^{2-5}$ revealed that the cryotrons can be potentially used as superconducting computer elements (switches, binary adders, shift registers, AND/OR gates, etc.). However the performance of such elements at high frequencies was found to be worse than ordinary semiconducting chips, since the cryotrons were rather slow and energy-consuming. Furthermore, the miniaturization of cryotron-like devices, which appears to be important for reducing the characteristic time constant, was very limited at those times.

Revival of interest to superconductor-electromagnet (S/Em) hybrids came in the 1990s in connection with the problem of the influence of a spatially modulated magnetic field on superconductivity. The development of advanced techniques for material deposition and lithographic methods have made it possible to fabricate composite structures with controlled arrangement of superconducting, normal metallic, ferromagnetic, and insulating layers at micron and submicron levels. Pannetier et $a l .{ }^{6}$ demonstrated that the dependence of the critical temperature $T_{c}$ on the applied magnetic field $H$ for such a hybrid system consisting of a plain $\mathrm{Al}$ film and a lithographically defined array of parallel metallic lines can be nonlinear and even nonmonotonous in contrast to a plain superconducting film in a uniform magnetic field. ${ }^{7}$ Such tunability of the $T_{c}(H)$ dependence by the stray field of the meander-like wire reflects directly the controllable modification of the standard Landau spectrum for the order parameter (OP) wave function in a periodic magnetic field. ${ }^{8-10}$ It is worth noting that keeping the nonuniform magnetic field of the control coils/wires requires expenditure of energy and may result in parasitic heating effects. Therefore, later on the interest was turned to superconductor-ferromagnet (S/F) hybrids since the inhomogeneous magnetic field in S/F systems, conditioned by the nonuniform distribution of magnetization, can be obtained without energy costs. The influence of a nonuniform magnetic field produced by ferromagnetic elements on nucleation of superconductivity and low-temperature properties of superconducting specimens was studied intensively for flux-coupled S/F hybrids during the last two decades (see Refs. 11-15 and references therein). We would like to note that the amplitude of the stray magnetic field and its profile are dictated by the saturated magnetization of the ferromagnet and by the shape of the ferromagnetic elements, which eventually restricts the flexibility of the S/F hybrids.

In the present paper we report on a so far unexplored limit of a cryotron-like system at micro- and nanoscales bringing together the ideas and approaches developed for both S/Em and S/F hybrids. With that purpose we fabricated a composite structure consisting of a type-II superconducting strip and a single straight current-carrying $\mathrm{S}$ wire oriented perpendicular to the strip. Since the stray magnetic field of the straight wire is maximal near the wire and it decays approximately as $1 / r$ at large distances $r$ from the wire, one can expect that only a small part of the superconducting strip in close vicinity to the wire will be affected by the nonuniform magnetic field. ${ }^{16}$ A simple change in the current in the wire, $I_{w}$, gives us the opportunity to control the maximal value of the magnetic field, $B_{0}$, generated by the current in the control wire. By varying $B_{0}$ one can create different states with partially and completely suppressed superconductivity in the considered cross-film cryotron. Thus, the main point of our research is to "scan" all possible intermediate states lying between uniform superconductivity (for small $B_{0}$ ) and fully depleted superconductivity (for large $B_{0}$ ) and to study the effect of the transitions between various superconducting states on the critical current for mesoscopic type-II cross-film cryotrons. 


\section{THEORY}

\section{A. Model}

To simulate the onset of vortex dynamics in the superconducting strip in the field of the current-carrying wire (i.e., in the cross-film cryotron) under the action of the transport current we use a time-dependent Ginzburg-Landau model. ${ }^{17}$ The thickness $D_{s}$ of the superconducting strip is assumed to be infinitely small, so the effective magnetic field penetration length $\Lambda=\lambda_{L}^{2} / D_{s}$ exceeds substantially the lateral dimensions of the superconducting sample $\left(\lambda_{L}\right.$ is the London penetration depth). Consequently, both the magnetic field $\mathbf{B}=\operatorname{rot} \mathbf{A}$ and the vector potential $\mathbf{A}$ are determined solely by external sources. We use the following units: $m^{*} \sigma_{n} \beta /\left(2 e^{2} \tilde{\alpha}\right)$ for time, the coherence length $\xi(0)$ at temperature $T=0$ for distances, $\Phi_{0} /[2 \pi \xi(0)]$ for the vector potential and $4 e \tilde{\alpha}^{2} \xi(0) /(\hbar \beta)$ for the current density, where $\alpha=-\tilde{\alpha} \tau$ and $\beta$ are the conventional parameters of the Ginzburg-Landau expansion, $\tau=\left(1-T / T_{c 0}\right), T_{c 0}$ is the critical temperature at $B=0, e$ and $m^{*}$ are the charge and the effective mass of carriers, $\sigma_{n}$ is the normal state conductivity. Then the time-dependent Ginzburg-Landau equations take the form $^{18}$

$$
\begin{aligned}
& u\left(\frac{\partial}{\partial t}+i \varphi\right) \psi=\tau\left(\psi-|\psi|^{2} \psi\right)+(\nabla+i \mathbf{A})^{2} \psi, \\
& \nabla^{2} \varphi=\operatorname{div} \mathbf{j}_{s}, \quad \mathbf{j}_{s}=-\frac{i}{2} \tau\left\{\psi^{*}(\nabla+i \mathbf{A}) \psi-\text { c.c. }\right\} .
\end{aligned}
$$

Here $\psi$ is the normalized order parameter (OP), $\varphi$ is the dimensionless electrical potential, $\mathbf{j}_{s}$ is the dimensionless density of superconducting currents, $u$ is the rate of the OP relaxation, c.c. stands for complex conjugate. To complete the problem we apply the boundary conditions

$$
\left(\frac{\partial}{\partial n}+i A_{n}\right)_{\Gamma} \psi=0, \quad\left(\frac{\partial \varphi}{\partial n}\right)_{\Gamma}=j_{\mathrm{ext}},
$$

which seems to be natural for the superconductor/vacuum or superconductor/insulator interfaces, $n$ is the normal vector to the sample's boundary $\Gamma, j_{\text {ext }}$ is the normal component of the inward (outward) flow of the external current. To detect a vortex on the grid cell corresponding to the certain grid node $\left(x_{i}, y_{j}\right)$, we use the following criterion: ${ }^{19}$

$$
\begin{aligned}
& \arg \left(\psi_{i, j}^{*} \psi_{i, j+1}\right)+\arg \left(\psi_{i, j+1}^{*} \psi_{i+1, j+1}\right) \\
& \quad+\arg \left(\psi_{i+1, j+1}^{*} \psi_{i+1, j}\right)+\arg \left(\psi_{i+1, j}^{*} \psi_{i, j}\right)=2 \pi N,
\end{aligned}
$$

$\psi_{i, j}=\psi\left(x_{i}, y_{j}\right), \arg (\psi)$ is the argument of the complex number $\psi_{i, j}$, belonging to the interval $(-\pi, \pi]$. If $N=+1$, there is a vortex in the cell, while $N=-1$ corresponds to an antivortex. It is important to note that we define the vortex (antivortex) as a position of the singularity of the OP phase, $\theta=\arg (\psi)$, however the direction, in which the superconducting currents circulate around the center of the vortex (antivortex), depends on the charge of carriers (i.e., the $e$ sign).

To facilitate further comparison between theory and experiment (Sec. III), for our modeling we choose the parameters, typical for mesoscopic Al-based superconductors: the coherence length $\xi(0)=0.15 \mu \mathrm{m}$ at $T=0$, length and width of the strip $L_{x}=9 \mu \mathrm{m}$ and $L_{y}=3 \mu \mathrm{m}$, width and thickness of the (a)
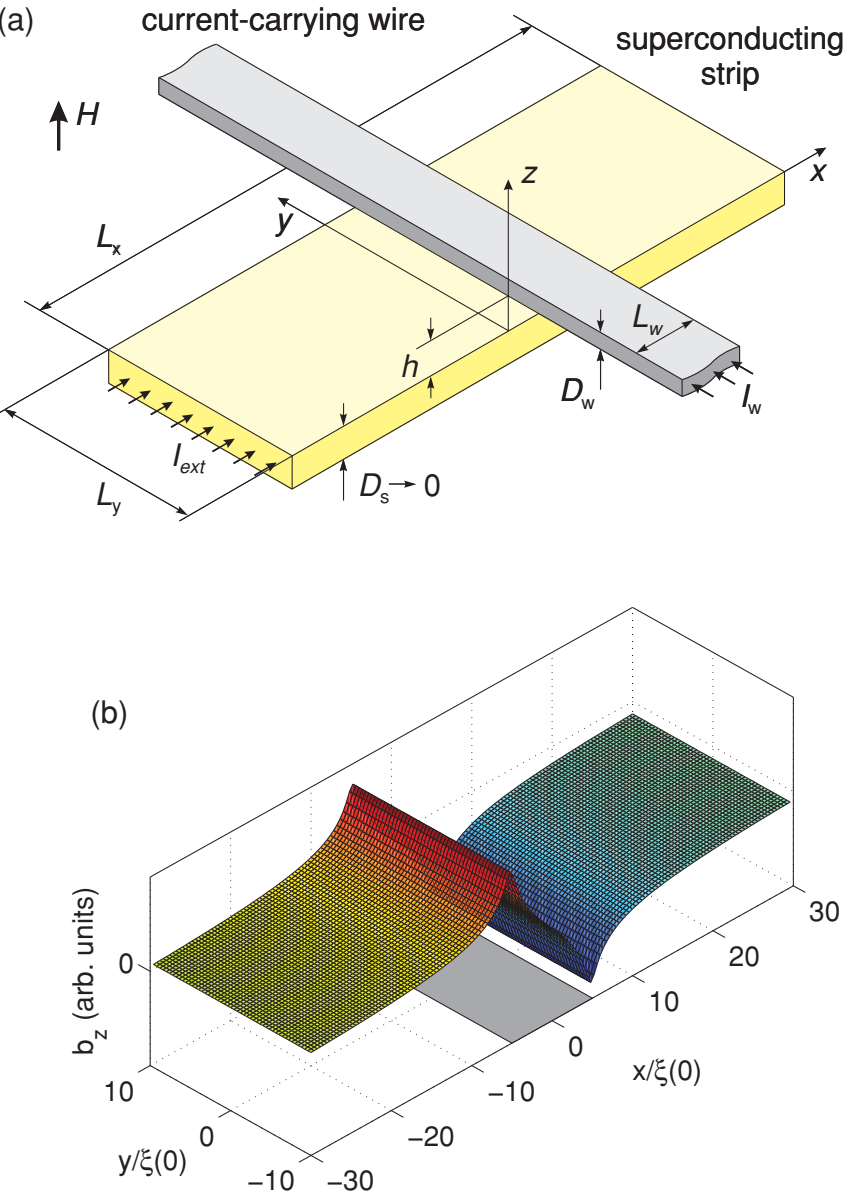

FIG. 1. (Color online) (a) Schematic presentation of the mesoscopic cross-film cryotron. (b) Profile of the $z$ component of the magnetic field, induced by the current-carrying wire, in the middle plane of the superconducting strip. The maximal and minimal $b_{z}$ values correspond to the edges of the current-carrying wire (shown in gray).

current-carrying wire $L_{w}=1.5 \mu \mathrm{m}$ and $D_{w}=0.05 \mu \mathrm{m}$, and the separation between the strip and the wire $h=0.05 \mu \mathrm{m}$ (Fig. 1).

\section{B. Mixed state in cross-film cryotron in equilibrium at $\boldsymbol{H}=\mathbf{0}$}

The stray field generated by the control wire is uniform across the superconducting bridge (in the $y$ direction, Fig. 1(b)). However in the $x$ direction the field is substantially inhomogeneous with the $b_{z}$ component reaching its extreme values near the edges of the current-carrying wire. The increase in the control current $I_{w}$ leads to a gradual enhancement of the maximal $b_{z}$ value. This eventually results in the local suppression of the energy barrier for vortex penetration from the edges of the strip near the $\left|b_{z}\right|$ maxima. These regions play the role of predefined gates for vortex entry.

The stray field of the control wire acts as (i) a tunable source of vortices and antivortices and, (ii) a vortex trap which prevents vortex-antivortex pairs from their mutual annihilation and the escape from the superconducting bridge. The latter follows from the distribution of the screening superconducting currents $j_{s}$ induced by the current-carrying wire [see Fig. 3(b)]. 

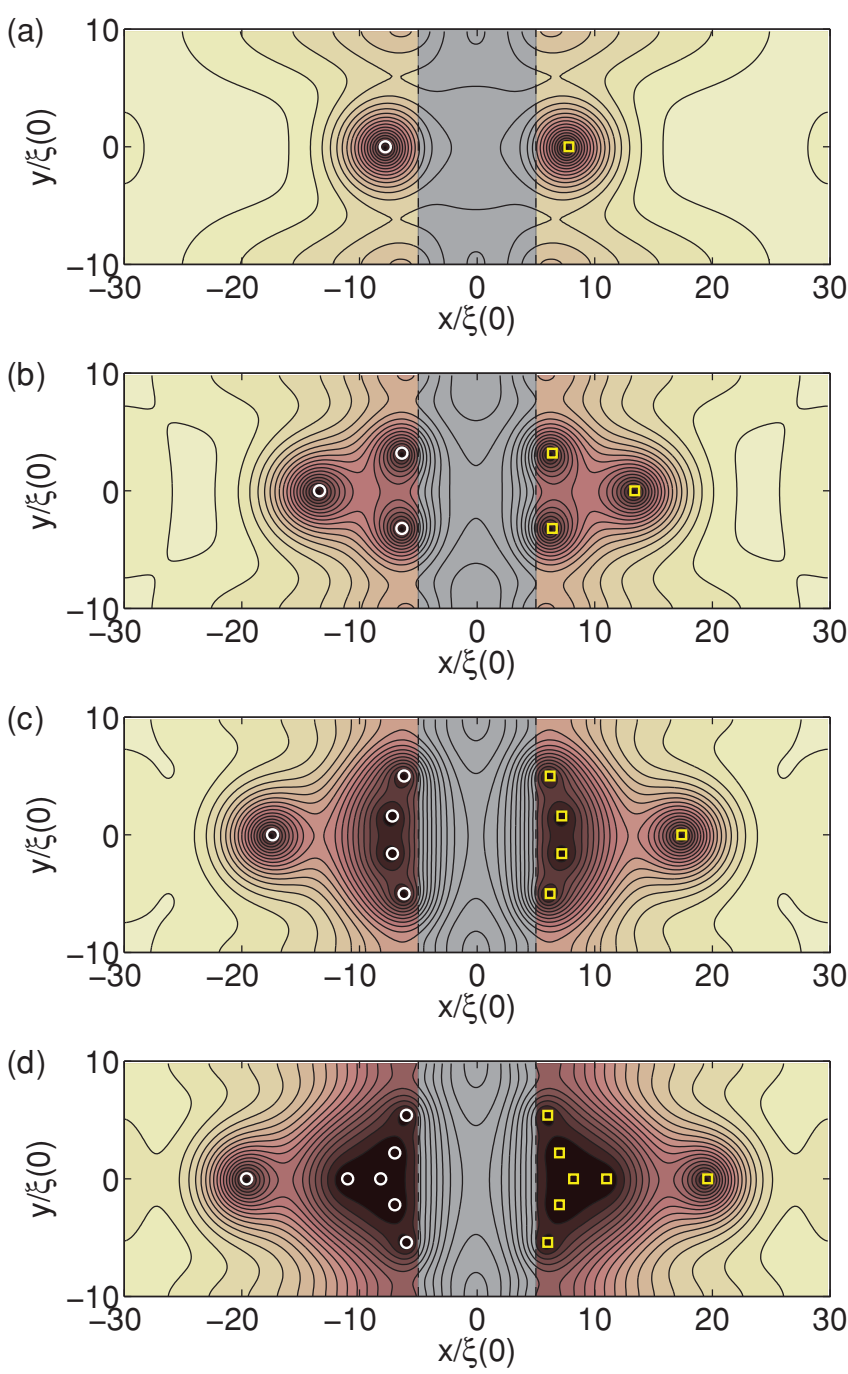

FIG. 2. (Color online) Contour plots of $|\psi|$, showing equilibrium vortex-antivortex patterns in the thin superconducting strip [60 $\xi(0) \times 20 \xi(0)$ in size], appearing at $H=0$ and $T=0.9 T_{c 0}$ in the field of the current-carrying wire: $I_{w}=5 \mathrm{~mA}(\mathrm{a}), I_{w}=8 \mathrm{~mA}$ (b), $I_{w}=10 \mathrm{~mA}$ (c), and $I_{w}=14 \mathrm{~mA}(\mathrm{~d})$. Lighter shades correspond to higher $|\Psi|$ values, darker shades are the regions with suppressed superconductivity. The grey rectangle in the center of the strip is the projection of the current-carrying wire. The symbols depict the OP phase singularities (i.e., the cores of the vortices and antivortices).

Indeed, the different signs of the $x$ component of $j_{s}$ near the top and bottom edges of the strip mean that the Lorentz force $\mathbf{f}_{L}=\left(\Phi_{0} / c\right)\left[\mathbf{j}_{s} \times \mathbf{z}_{0}\right]$ acting on the vortex (antivortex) near these edges will be oriented in such a way in order to push the vortex (antivortex) to the inner part of the strip. In the absence of an external magnetic field $H$ the appearance of a vortex should be accompanied by the formation of a symmetrically positioned antivortex and the number of vortex-antivortex pairs $N$ increases as $\left|I_{w}\right|$ increases (Fig. 2). Since the $b_{z}$ field roughly decays inversely proportional to the distance from the wire, the vortices and antivortices are also distributed nonuniformly: the closer to the wire, the smaller the distance between two vortices (antivortices) is.

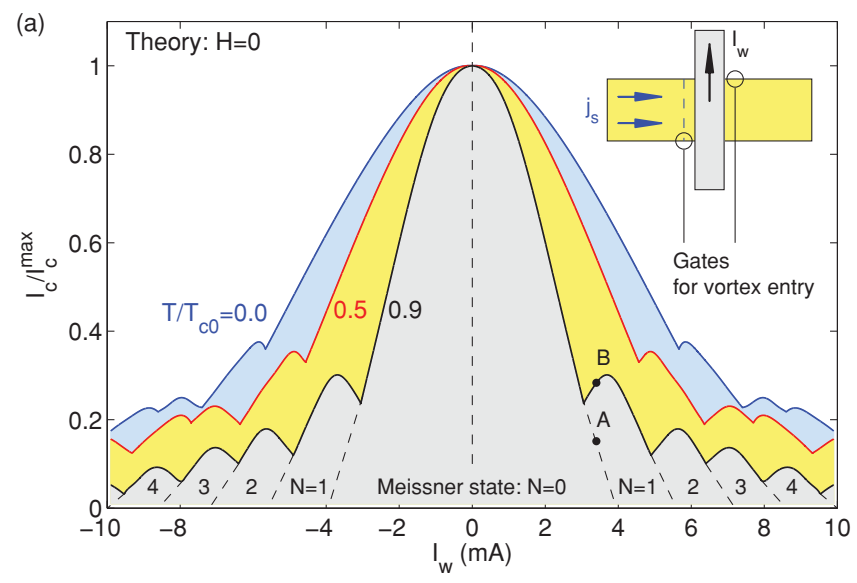

(b) $|\psi(x, y)|$ at point $\mathrm{A}$

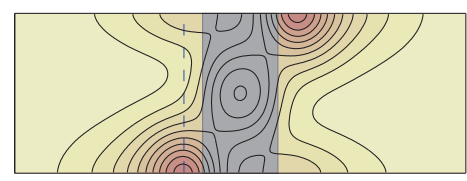

Cross-sectional analysis

(c) $|\psi(x, y)|$ at point B
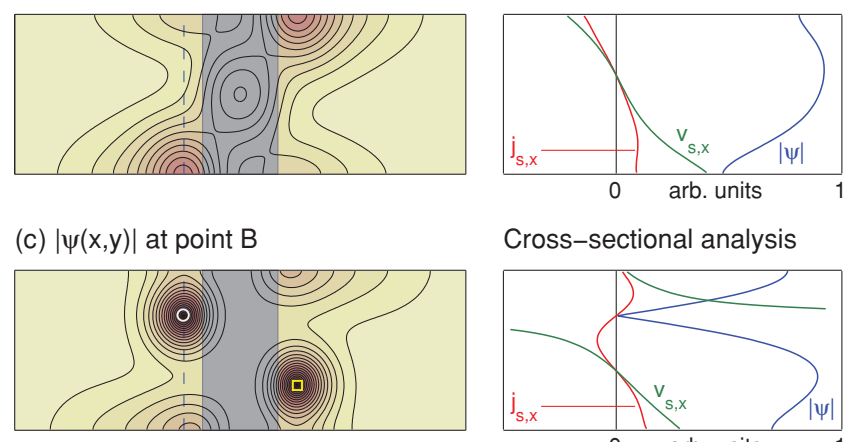

Cross-sectional analysis

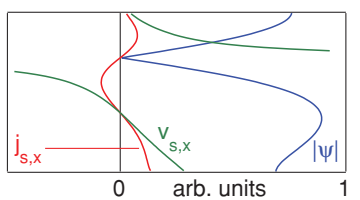

FIG. 3. (Color online) (a) Critical current $I_{c}$ as a function of control current $I_{w}$ calculated at $H=0$ and $T / T_{c 0}=0,0.5$, and 0.9. For each segment of the $I_{c}-I_{w}$ diagram we indicate the number of the trapped vortices/antivortices $N$ in the strip. (b),(c) Left panels show the stationary OP wave functions $|\psi|$ calculated for $T / T_{c 0}=$ 0.9 and $I_{w}=3.4 \mathrm{~mA}$ for two different values of the injected current [points A and B in (a)]. The gray rectangle in the center of the strip is the projection of the current-carrying wire. The correspondent right panels show the profiles of the order parameter wave function $|\psi|$, the longitudinal $x$ components of the current $j_{s, x}$ and the supercurrent velocity $v_{s, x} \propto\left(\partial \theta / \partial x-2 \pi A_{x} / \Phi_{0}\right)$ across the strip (i.e., along the dashed line).

\section{Critical current of cross-film cryotron at $H=0$}

Depending on the magnitude of the bias current $I_{\text {ext }}$, injected into the superconducting strip, there are two distinct regimes. After applying a small bias current the disturbed vortex and antivortex tend to relax into a new stationary configuration. This state with motionless vortex-antivortex pairs is characterized by the absence of an electrical field inside the superconductor (except for tiny regions near the boundaries where the current injection takes place) and by a zero voltage drop. If $I_{\text {ext }}$ exceeds the critical current $I_{c}$, the stability of the vortex-antivortex ensemble breaks down: vortex and antivortex periodically in time enter and exit into/from the superconductor. This means that the sample has switched to a dissipative regime. The calculated dependencies $I_{c}\left(I_{w}\right)$ at $H=0$ for different temperatures are shown in Fig. 3(a). We see that $I_{c}$ decays with oscillations as $I_{w}$ sweeps and the $I_{c}$ oscillations become more pronounced at high temperatures. 
We can explain the appearance of the $I_{c}\left(I_{w}\right)$ oscillations as follows. If $H=0$ and $I_{w}=0$, superconductivity will survive until the injected current density exceeds the depairing limit. For nonzero $I_{w}$ the transition from the nondissipative state to the resistive regime occurs via permanent formation of vortexantivortex pairs at the opposite edges of the superconducting bridge and their motion across the bridge (see inset in Fig. 3). Due to the superposition of the injected current and the currents, induced by the wire, the limit for the vortex-antivortex pair generation can be reached at smaller values of the bias current; therefore $I_{c}$ monotonously decreases as $I_{w}$ increases. A further increase in $I_{w}$ leads to (i) a decrease in the threshold value of the bias current required for the creation of the first vortex, and (ii) an enhancement of the trapping potential for vortices. Both consequences make it possible to stabilize the vortex-antivortex pair in the presence of the transport current [Fig. 3(c)]. The self-currents generated by the vortex and antivortex partly compensate the superflow conditioned by the bias current near the regions with suppressed OP wave function. Therefore these "gates" will be closed and the formation of new vortices and antivortices will be impeded unless the maximal value for supercurrent velocity $\mathbf{v}_{s}$, which is proportional to $\nabla \theta-2 \pi \mathbf{A} / \Phi_{0}$, again reaches its critical value at the strip edges. This means that after the first vortexantivortex pair is trapped, the critical current will be larger than that in the Meissner state. Then this process is repeated periodically as $I_{w}$ increases, resulting in periodic variations in $I_{c}\left(I_{w}\right)$. Thus, the discrete change in the number of the pinned vortices and antivortices in the superconducting strip of a finite width can be identified by considering the position of the cusps on the $I_{c}\left(I_{w}\right)$ curve. In contrast to the $I_{c}$ oscillations observed in mesoscopic superconducting squares in a uniform magnetic field ${ }^{20}$ the nonuniform magnetic field gives us the possibility to see the quantization effects in a long superconducting strip, due to the confinement potential along the strip produced by the control wire.

The absolute value of the magnetic flux $\Phi_{1 / 2}$ piercing a half of the superconducting strip,

$$
\Phi_{1 / 2}=\int_{0}^{L_{x} / 2} \int_{-L_{y} / 2}^{L_{y} / 2}\left|b_{z}(x, y)\right| d x d y,
$$

is a linear function of $I_{w}$. In a certain sense the oscillations of the critical current of the cryotron as $I_{w}$ (or $\Phi_{1 / 2}$ ) varies, are similar to the standard Fraunhofer pattern describing the dependence of the critical current of a Josephson junction on the total magnetic flux $\Phi$ through the junction area. ${ }^{21,22}$ The built-in field of the wire guarantees reversible entrance of vortices and antivortices into the superconducting strip as $I_{w}$ increases, and the tunable modification of the distribution of the OP phase in the restricted part of the strip by the trapped vortices and antivortices. This area with partly suppressed superconductivity near the control wire acts like an effective weak link, since the vortex dynamics in this area determines the flow of the bias current and the resistance of the entire strip.

\section{Mixed state and critical current of cross-film cryotron at $\boldsymbol{H} \neq \mathbf{0}$}

The external magnetic field $H$, applied perpendicularly to the sample's plane, breaks the symmetry between vortex and
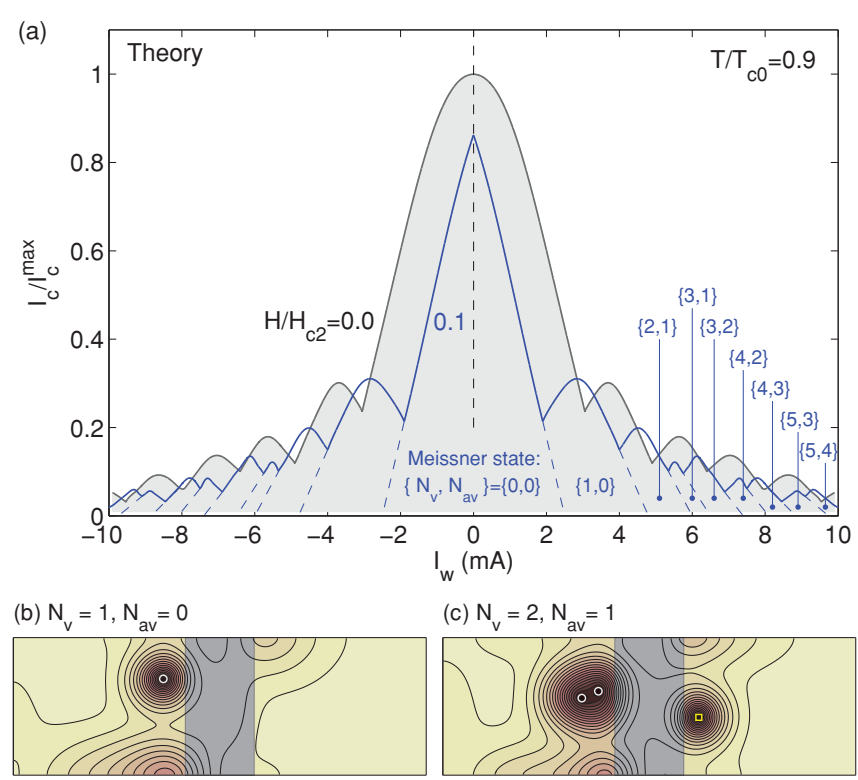

(c) $\mathrm{N}_{\mathrm{v}}=2, \mathrm{~N}_{\mathrm{av}}=1$
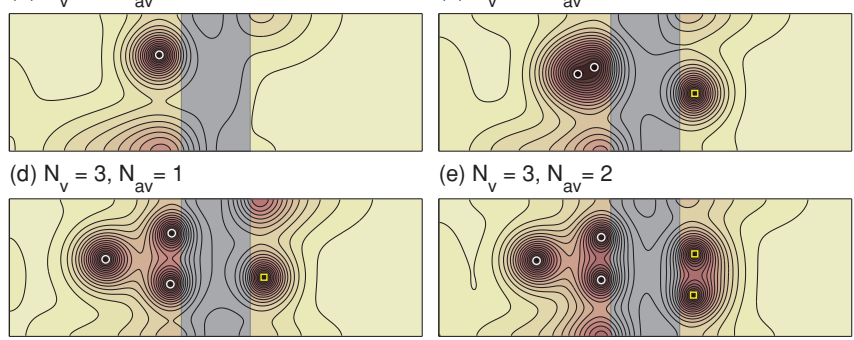

(e) $\mathrm{N}_{\mathrm{v}}=3, \mathrm{~N}_{\mathrm{av}}=2$

FIG. 4. (Color online) (a) The calculated dependencies $I_{c}\left(I_{w}\right)$ for $T / T_{c 0}=0.9$ and $H=0$ (black line) and $H / H_{c 2}=0.1$ (blue line). For each segment of the $I_{c}-I_{w}$ diagram we indicate the number of vortices $N_{v}$ and antivortices $N_{a v}$, trapped in the superconducting strip. (b-e) Examples of the stationary OP patterns at $T / T_{c 0}=0.9$ and $H / H_{c 2}=0.1$ and at bias current close to the corresponding critical values.

antivortex since the total magnetic flux piercing the sample is not zero anymore. As a result, the entrance of a new vortex is not generally accompanied by the entrance of an antivortex: the numbers of trapped vortices and antivortices, $N_{v}$ and $N_{a v}$, may differ in contrast to the previously considered case $H=0$.

The results of the calculations for $T / T_{c 0}=0.9$ and at $H / H_{c 2}=0$ and $H / H_{c 2}=0.1$ are compared in Fig. 4(a). It is clear that the oscillating behavior in the $I_{c}$ vs. $I_{w}$ remains even in the presence of the applied magnetic field. Since the constructive superposition of (i) the injected current, (ii) the currents induced by the wire and (iii) the currents induced by the external field, enhances the local flow of superconducting condensate, the conditions for vortex entry will be fulfilled at smaller $I_{w}$ values. This results in a shift of all the cusps on the $I_{c}\left(I_{w}\right)$ dependence toward to zero. The nonequivalence between vortices and antivortices allows us to get new stable states of reduced symmetry [Figs. 4(b)-4(e)] which are forbidden for the symmetrical sample at $H=0$. The transition between such states sweeping $I_{w}$ are accompanied by additional cusps, which leads to more complex oscillatory properties of the $I_{c}$ on $I_{w}$ dependence.

\section{EXPERIMENT AND DISCUSSION}

To study the transport properties of the mesoscopic crossfilm cryotron, we fabricated the hybrid structures consisting of a $4 \mu \mathrm{m}$ wide and $120 \mathrm{~nm}$ thick superconducting $\mathrm{Al}$ strip 
on top of a $1.5 \mu \mathrm{m}$ wide and $50 \mathrm{~nm}$ thick current-carrying $\mathrm{Nb}$ wire. The $\mathrm{Nb}$ thin film was deposited via dc magnetron sputtering at a rate of $2.5 \mathrm{~nm} / \mathrm{s}$ on an oxidized Si substrate at room temperature under the pressure of Ar plasma of $6 \times$ $10^{-3}$ mbar. The $\mathrm{Nb}$ wire was fabricated by e-beam lithography in combination with argon ion milling. The Al film was grown in a molecular-beam epitaxy apparatus at a rate of $0.2 \mathrm{~nm} / \mathrm{s}$ at room temperature and nominal pressure of $1 \times 10^{-8}$ mbar. The Al strip was patterned by standard e-beam lithography, subsequent evaporation and lift-off technique. A $120 \mathrm{~nm}$ thick Ge layer separates the two metals from each other to avoid electrical contact between them. We tested more than 30 identical hybrid samples prepared at different times but under similar conditions. At least ten samples have no problems with electrical leakage, since the applying a voltage between $\mathrm{Al}$ strip and $\mathrm{Nb}$ wire leads to an absence of detectable current. It gives us a lower limit on the contact resistance of the order of $5 \mathrm{M} \Omega$. An atomic force microscopy (AFM) image of the sample is shown in Fig. 5.

Considering the dependence of the sample resistance $R$ on $H$ and its displacement upon varying $T$, one can compose the phase transition line $T_{c}(H)$ shown in Fig. 6(b). By identifying this line with the temperature dependence of the critical field of surface superconductivity $H_{c 3}=1.69 H_{c 2}^{(0)}\left(1-T / T_{c 0}\right)$, we estimated $T_{c 0} \simeq 1.265 \mathrm{~K}$, the upper critical field $H_{c 2}^{(0)} \simeq$ $104 \mathrm{Oe}$, and the coherence length $\xi(0)=\left(\Phi_{0} / 2 \pi H_{c 2}^{(0)}\right)^{1 / 2} \simeq$ $175 \mathrm{~nm}$ extrapolated to $T=0$. The mean free path $\ell \simeq 35 \mathrm{~nm}$ can be obtained from the value of the normal resistivity $\rho_{n}$ at low temperatures according to the formula: ${ }^{23} \rho_{n} \ell \simeq$ $6 \times 10^{-16} \Omega \cdot \mathrm{m}^{2}$. The $\ell \sim 35 \mathrm{~nm}$ value gives us the London penetration depth and the effective magnetic field penetration depth $\lambda_{L}(0) \simeq 175 \mathrm{~nm}$ and $\Lambda(0)=\lambda_{L}^{2}(0) / D_{s} \simeq 250 \mathrm{~nm}$ respectively in dirty limit at $T=0$. All obtained values are in accordance with previous studies on thin film Al structures. ${ }^{24}$

Figure 7(a) shows the $I_{c}$ of the Al strip as a function of the current $I_{w}$ in the control $\mathrm{Nb}$ wire, measured at $H=0$

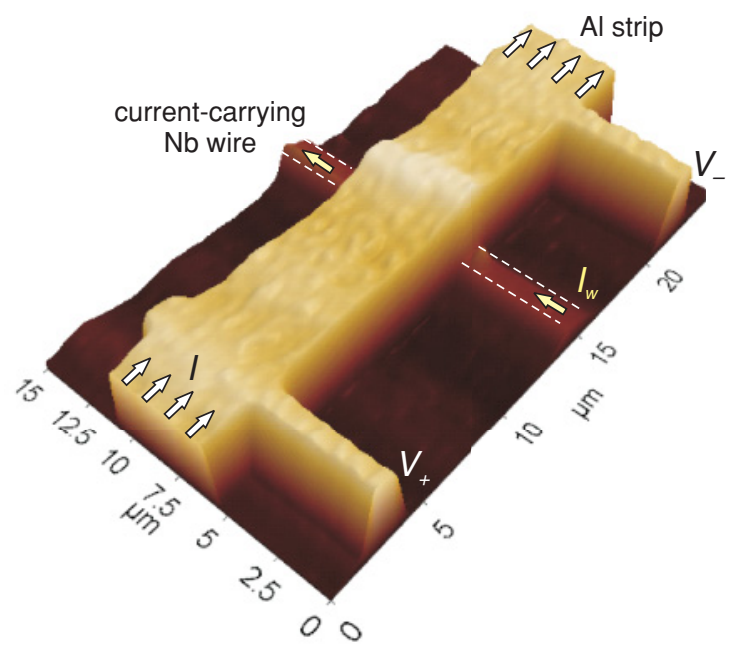

FIG. 5. (Color online) AFM image of the sample: the top (light) element is the superconducting Al strip with two perpendicular contacts for the measurement of the voltage drop, the bottom element oriented perpendicular to the strip is the current-carrying $\mathrm{Nb}$ wire.
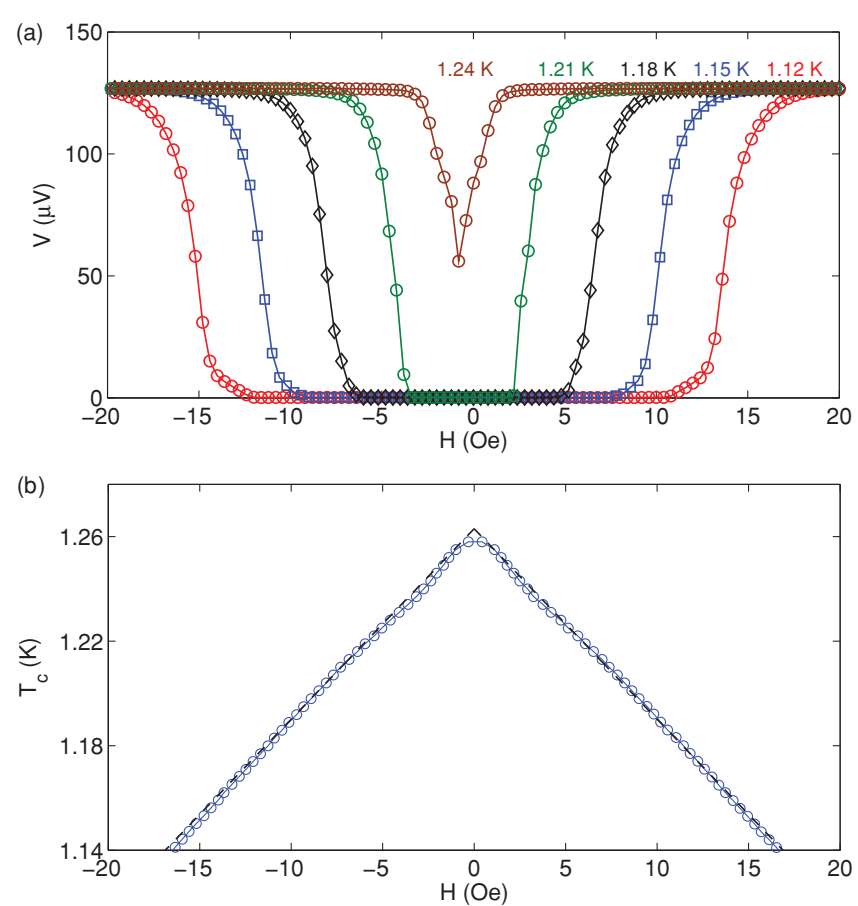

FIG. 6. (Color online) (a) Typical dependence of the voltage drop, $V$, as a function of $H$ at $I_{w}=0$ for different temperatures. The bias dc current $I$ is equal to $50 \mu \mathrm{A}$, the normal state resistance $R_{n} \simeq 2.54 \Omega$. (b) The phase boundary $T_{c}(H)$ extracted from the magnetoresistive measurements at $I_{w}=0$ according to the criterion $R(H, T)=0.99 R_{n}$. The dashed line is the linear approximation $\left(1-T / T_{c 0}\right)=0.59|H| / H_{c 2}^{(0)}$, where $H_{c 2}^{(0)} \simeq 104 \mathrm{Oe}, T_{c 0}=1.265 \mathrm{~K}$.

and different temperatures close to $T_{c 0}$. Experimentally the critical current was determined from the isothermal current $(I)$-voltage $(V)$ dependencies for different voltage criteria (from $0.1 \mu \mathrm{V}$ up to $10 \mu \mathrm{V}$ between the two voltage contacts at a distance $20 \mu \mathrm{m}$ ) for the measured voltage drop. ${ }^{25}$ For our measurements the accuracy $\delta I_{c}$ of the determination of the critical current was $3 \mu \mathrm{A}$. This means that within the interval $2 \delta I_{c}$ we observed a sharp transition from the superconducting state (typical noise level of the order of $5 \times 10^{-8} \mathrm{~V}$ ) to the resistive state. The plateau in $I_{c}\left(I_{w}\right)$ might be associated with the influence of sample imperfections, which facilitate the formation of vortices near defects even in zero magnetic field at current densities smaller than the theoretical limit. In accordance with our simulations the monotonously decreasing part of $I_{c}\left(I_{w}\right)$ has to be attributed to the transition from the Meissner state to the resistive state with a single moving vortex-antivortex pair. The next stages correspond to the stabilization of the first and all subsequent vortex-antivortex pairs in the sample. The switching between these states at varying $I_{w}$ corresponds to the local $I_{c}$ minima. We clearly observed the reproducible oscillations of the critical current for all measured samples.

The effect of the external field is illustrated by Fig. 7(b). We observed the anticipated shift of the monotonously decreasing part in $I_{c}\left(I_{w}\right)$ toward smaller $I_{w}$ values, conditioned by the constructive superposition of the injected current, the currents induced by the wire and by the external field. This 

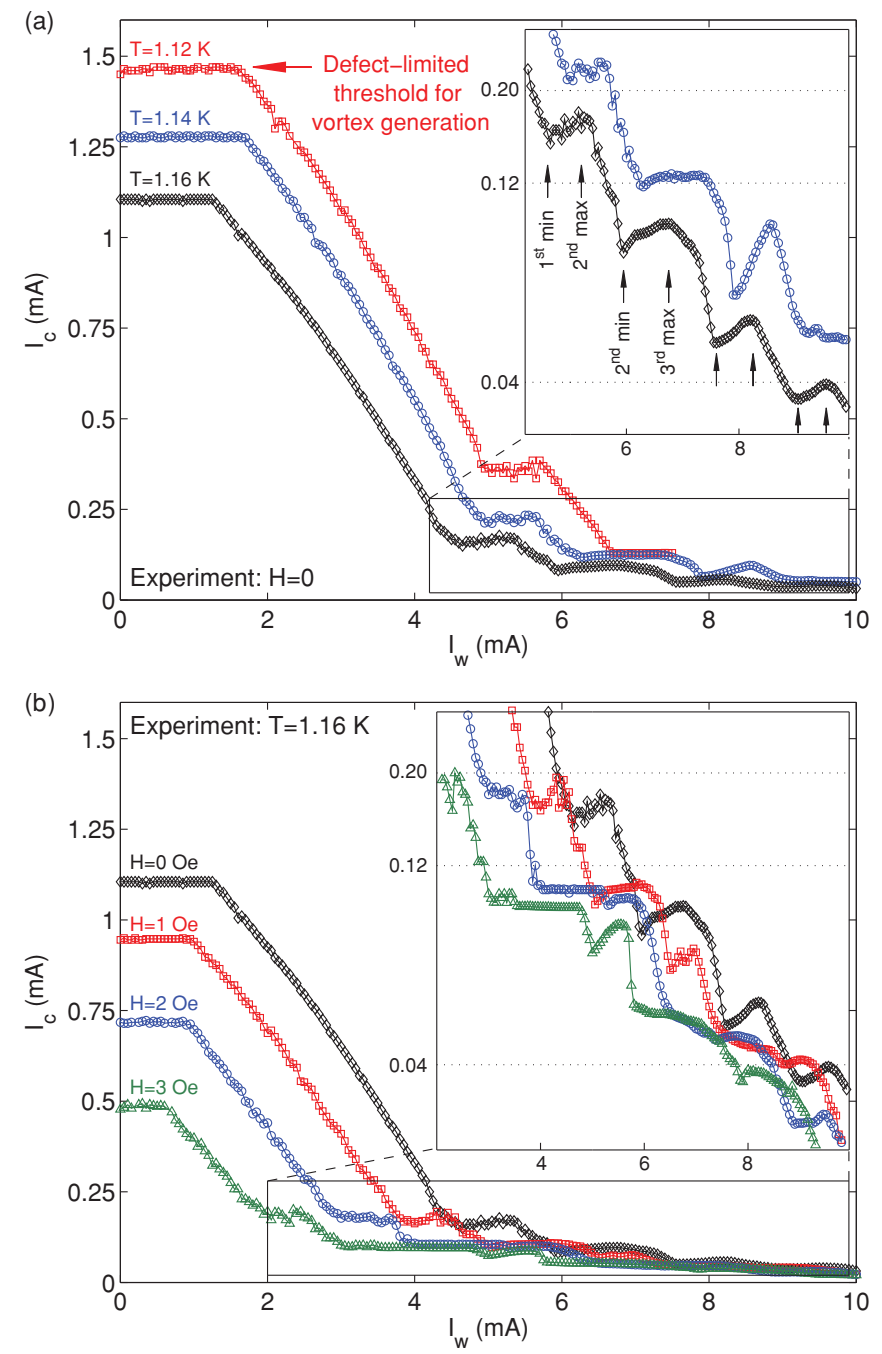

FIG. 7. (Color online) (a) Measured dependence $I_{c}$ on $I_{w}$ at $H=$ 0 for different temperatures. (c) Measured dependence $I_{c}$ on $I_{w}$ at $T=1.16 \mathrm{~K}$ for different $H$ values. Both insets in (b) and (c) show zoomed parts of the $I_{c}\left(I_{w}\right)$ curves (within the box) in semilogarithmic scale. The arrows in the inset (a) show the positions of the extremal $I_{c}$ values for $T=1.16 \mathrm{~K}$ and $H=0$, which are compared with theory in Fig. 8. The confidence intervals are smaller than the size of symbols, and therefore error bars cannot be shown in this scale.

ensures that the conditions for the first vortex entry will be fulfilled at smaller $I_{w}$ values. It is interesting to note that the shape of the oscillating $I_{c}\left(I_{w}\right)$ curves becomes more complicated as $H$ increases. We suppose that the externalfield-induced modification of $I_{c}\left(I_{w}\right)$ reflects the formation of exotic nonsymmetrical vortex states and their depinning under the action of the bias current.

To compare theory and experiment, we plot the positions of the minima and maxima in the $I_{c}\left(I_{w}\right)$ dependence at $H=0$ (Fig. 8). It is easy to see that our simple model describes both the general oscillating behavior for the $I_{c}\left(I_{w}\right)$ dependence for real sample and the observed period $\Delta I_{w}$ quite well. The fact that all theoretical points in Fig. 8 lie below the experimental data, may be caused, e.g., by a nonuniform current distribution in the control wire, or by a small difference between the
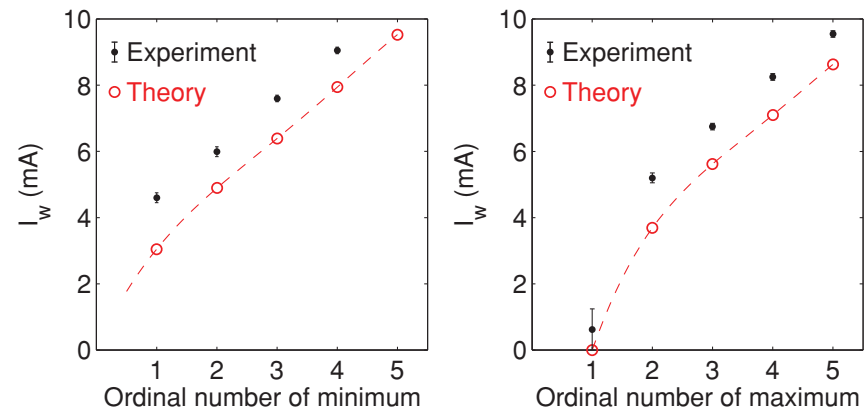

FIG. 8. (Color online) Positions of the $I_{c}$ minima (left panel) and the $I_{c}$ maxima (right panel), calculated at $H=0$ and $T / T_{c 0}=0.9$ (red circles) and measured at $T=1.16 \mathrm{~K}\left(T / T_{c 0} \simeq 0.91\right.$, black dots). Dashed red lines are guide to the eyes. The positions of the maxima and minima determined experimentally are also marked by vertical arrows in the inset of Fig. 7(a).

parameters of the model problem and the dimensions of the tested samples. Nevertheless using the estimated period $\Delta I_{w} \simeq 1.45 \mathrm{~mA}$, one can calculate the change in the magnetic flux $\Delta \Phi_{1 / 2}$ through the half of the superconducting strip and corresponding to the appearance of the vortex-antivortex pair: $\Delta \Phi_{1 / 2} \simeq 1.1 \Phi_{0}$.

In this paper we have reported on the first results obtained for the prepared S/Em samples. We note that the oscillatory behavior of the critical current as a function of $I_{w}$ was found to be reproducible for all measured samples, which had identical geometry and size as the presented device. However the issues concerning the influence of the dimensions of the superconducting strip and the current-carrying wire on the $I_{c}$ oscillations remain unaddressed.

\section{CONCLUSION}

We studied the transport properties of a hybrid system consisting of a superconducting strip and a current-carrying wire, oriented perpendicular to the strip. The stray field, generated by the control wire, makes it possible to tune the transport properties of the long superconducting strip locally by means of creation and pinning of vortices and antivortices. This area, where vortices and antivortices become confined, plays the role of a "bottleneck" for the transport current, since the motion of vortices and antivortices is the main source of the dissipation. Thus, the peculiar configurations of vortex-antivortex pairs determine the critical current $I_{c}$ of the $\mathrm{S}$ strip. The transition between different stable vortex patterns upon varying $I_{w}$ results in an oscillatory dependence of $I_{c}$ on $I_{w}$. The observed effect, which looks similar to the Fraunhofer oscillating pattern for conventional Josephson junctions, can be potentially interesting for the design of superconducting interferometer devices based on S/F or S/Em hybrid structures.

\section{ACKNOWLEDGMENTS}

This work was supported by the Methusalem Funding of the Flemish Government, the NES - ESF program, the Belgian IAP, the Carl-Zeiss-Stiftung, and the Deutsche 
Forschungsgemeinschaft (DFG) via the SFB/TRR 21, the Russian Fund for Basic Research, RAS under the Program "Quantum physics of condensed matter" and Federal Target
Program "Scientific and educational personnel of innovative Russia in 2009-2013." A.V.S, W.G., and J.V.d.V. acknowledge support from F.W.O.
${ }^{1}$ D. A. Buck, Proc. IRE 44, 482 (1956).

${ }^{2}$ P. G. de Gennes, Superconductivity of Metals and Alloys (W. A. Benjamin Inc., New York, 1966).

${ }^{3}$ J. W. Bremer, Superconductive Devices (McGraw-Hill, New York, 1962).

${ }^{4}$ J. M. Lock, Rep. Prog. Phys. 25, 37 (1962).

${ }^{5}$ V. L. Newhouse, in Superconductivity, edited by R. D. Parks (Marcel Dekker, New York, 1969), Vol. 2, Chap. 22.

${ }^{6}$ B. Pannetier, S. Rodts, J. L. Genicon, Y. Otani, and J. P. Nozieres, in Macroscopic Quantum Phenomena and Coherence in Superconducting Networks, edited by C. Giovannella and M. Tinkham (World Scientific, Singapore, 1995), pp. 17-24.

${ }^{7}$ See e.g., M. Tinkham, Introduction to Superconductivity, 2nd ed. (McGraw-Hill, New York, 1996).

${ }^{8}$ F. M. Peeters and P. Vasilopoulos, Phys. Rev. B 47, 1466 (1993); I. S. Ibrahim and F. M. Peeters, ibid. 52, 17321 (1995).

${ }^{9}$ A. Yu. Aladyshkin, A. I. Buzdin, A. A. Fraerman, A. S. Mel'nikov, D. A. Ryzhov, and A. V. Sokolov, Phys. Rev. B 68, 184508 (2003).

${ }^{10}$ A. Yu. Aladyshkin and V. V. Moshchalkov, Phys. Rev. B 74, 064503 (2006).

${ }^{11}$ M. Lange, M. J. Van Bael, Y. Bruynseraede, and V. V. Moshchalkov, Phys. Rev. Lett. 90, 197006 (2003).

${ }^{12}$ Z. R. Yang, M. Lange, A. Volodin, R. Szymczak, and V. V. Moshchalkov, Nature Materials 3, 793 (2004).

${ }^{13}$ I. F. Lyuksyutov and V. L. Pokrovsky, Adv. Phys. 54, 67 (2005).
${ }^{14}$ M. Vélez, J. I. Martín, J. E. Villegas, A. Hoffmann, E. M. Gonzalez, J. L. Vicent, and I. K. Schuller, J. Magn. Magn. Mater. 320, 2547 (2008).

${ }^{15}$ A. Yu. Aladyshkin, A. V. Silhanek, W. Gillijns, and V. V. Moshchalkov, Supercond. Sci. Technol. 22, 053001 (2009).

${ }^{16} \mathrm{~A}$ similar effect of the stray magnetic field for the S/F. bilayers was considered by E. B. Sonin, Sov. Tech. Phys. Lett. 14, 714 (1988).

${ }^{17}$ B. I. Ivlev and N. B. Kopnin, Usp. Fiz. Nauk 142, 435 (1984); Adv. Phys. 33, 47 (1984).

${ }^{18}$ A. V. Kapra, V. R. Misko, D. Y. Vodolazov, and F. M. Peeters, Supercond. Sci. Technol. 24, 024014 (2011).

${ }^{19}$ A. S. Mel'nikov, I. M. Nefedov, D. A. Ryzhov, I. A. Shereshevskii, V. M. Vinokur, and P. P. Vysheslavtsev, Phys. Rev. B 65, 140503(R) (2002).

${ }^{20}$ See e.g., D. Yu. Vodolazov, F. M. Peeters, M. Morelle, and V. V. Moshchalkov, Phys. Rev. B 71, 184502 (2005).

${ }^{21}$ A. Barone and G. Paterno, Physics and Applications of the Josephson effect (John Wiley \& Sons, New York, 1982).

${ }^{22}$ A. A. Abrikosov, Fundamentals of the Theory of Metals (NorthHolland, Amsterdam, 1988).

${ }^{23}$ F. R. Fickett, Cryogenics 11, 349 (1971).

${ }^{24}$ W. Gillijns, A. Yu. Aladyshkin, A. V. Silhanek, and V. V. Moshchalkov, Phys. Rev. B 76, 060503(R) (2007).

${ }^{25}$ A. M. Campbell and J. E. Evetts, Critical Currents in Superconductors (Taylor \& Francis Ltd., London, 1972). 